\title{
Current adding transmission lines for compact MA-class linear transformer drivers
}

\author{
P.-A. Gourdain $\odot,{ }^{1,2,3 *}$ M. B. Adams $\odot,{ }^{1,4}$ M. Evans ${ }^{1,2}$ H. R. Hasson, ${ }^{1}$ R. V. Shapovalov, ${ }^{1,2}$ \\ R. B. Spielman, ${ }^{2}$ J. R. Young, ${ }^{1,2}$ and I. West-Abdallah ${ }^{1}$ \\ ${ }^{1}$ Department of Physics and Astronomy, University of Rochester, Rochester, New York 14627, USA \\ ${ }^{2}$ Laboratory for Laser Energetics, University of Rochester, Rochester, New York 14627, USA \\ ${ }^{3}$ Princeton Plasma Physics Laboratory (PPPL), Princeton University, Princeton, New Jersey 08543, USA \\ ${ }^{4}$ Flash Center, University of Chicago, Chicago, Illinois 60637, USA
}

(Received 23 October 2019; accepted 28 February 2020; published 30 March 2020; corrected 9 March 2021)

Linear transformer drivers can be used as fast pulsed-power generators to produce and study matter under extreme conditions, with densities larger than 10 times that of solids. While its scientific investigations can start in a laboratory, it will eventually require a source of $\mathrm{x}$ rays that can be provided only by specialized light source facilities. When relocation becomes a necessity, a compact, modular design is clearly advantageous. This paper shows how nested transmission lines can reduce considerably the footprint of MA-class pulsed-power generators based on linear transformer driver technology.

DOI: 10.1103/PhysRevAccelBeams.23.030401

\section{INTRODUCTION}

While linear transformer driver (LTD) [1-3] systems have been around for decades [4], new designs using fast $\left(t_{\text {rise }} \sim 100 \mathrm{~ns}\right)$, high-voltage $(V \sim 100 \mathrm{kV})$ capacitors make them a competitive alternative to Marx-bank generators. Further improvements would allow LTDs to deliver terawatts $[5,6]$ or even a petawatt [7] of power to an experimental load. A basic LTD cavity is presented in Fig. 1. In this design, fast capacitors are connected to a spark gap switch and are typically charged up to $\pm 100 \mathrm{kV}$. Together with the switch, they form the elementary unit, or brick (highlighted in Fig. 1). All bricks are laid inside a metal casing, forming a cavity. The cavity holds a set of magnetic cores to limit the current flowing inside the casing. Modern cavities typically deliver up to 1 MA and are fairly compact, with diameters slightly over $2 \mathrm{~m}$ [8]. Given a well-defined experimental goal, the voltage (V) required to drive a current (I) sufficient to magnetically compress a purely inductive load goes as

$$
V=L \frac{d I}{d t}
$$

This voltage can be estimated readily using

\footnotetext{
*on leave at PPPL.

Published by the American Physical Society under the terms of the Creative Commons Attribution 4.0 International license. Further distribution of this work must maintain attribution to the author(s) and the published article's title, journal citation, and DOI.
}

$$
V(\mathrm{MV})=\frac{L(\mathrm{nH}) I(\mathrm{MA})}{t(\mathrm{~ns})}
$$

For instance, initiating fusion reactions [9] using a fast $\mathrm{Z}$ pinch [10] with a load inductance on the order of $10 \mathrm{nH}$ requires a current of $10 \mathrm{MA}$ with a current rise time of $100 \mathrm{~ns}$. Under these conditions, the peak voltage will be at least $1 \mathrm{MV}$. Because of capacitor limitations $(100 \mathrm{kV} / 100 \mathrm{nF} /$ $50 \mathrm{kA}$ ), it is necessary to connect the cavities in series to

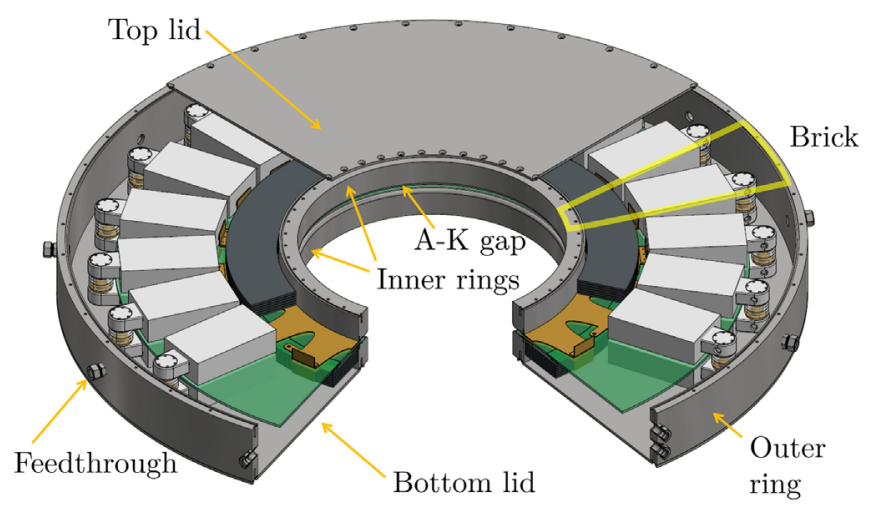

FIG. 1. A view of one of the cavities of HADES. Twenty-two bricks (composed of one spark gap switch and two capacitors) are connected to the inner rings via a set of strip lines (brass color). Two saturable cores (black) preclude the current from flowing inside the lid of the cavity. The inner rings and their anodecathode (A-K) gap are surrounding the nested transmission lines, shown in Fig. 2. The insulator between the top and bottom inner rings (green) is the only electrical break between both sides of the A-K gap. 
reach $1 \mathrm{MV}$, and then in parallel, to deliver $10 \mathrm{MA}$ to the load. This design requires two kinds of transmission line [11]. The first kind connects cavities together, forming a module. The second kind connects the modules to the load. In fast Marx-bank designs, the module transmission lines are replaced by pulse-forming lines and intermediate switches, required to compress a microsecond current pulse down to $100 \mathrm{~ns}$.

While smaller pulsed-power drivers $\left(I_{\text {peak }} \sim 1 \mathrm{MA}\right)$ cannot reach nuclear fusion conditions, they often target more fundamental problems, studying scale-invariant phenomena to benchmark astrophysical codes [12] or producing $\mathrm{x}$-ray sources [13] used in material studies. At low peak currents, the plasma can be diagnosed relatively well using a visible laser [14], a very attractive proposition when larger currents require $\mathrm{x}$-ray diagnostics. With a reduced number of modules, the transmission line geometry can be greatly simplified. This paper studies three different transmission line geometries, modeling transmission lines using an equivalent RLC circuit and comparing the results to a full three-dimensional electromagnetic code. While current rise time differs slightly between the two models, it is necessary to use the electromagnetic code to compute the strength of the electric field inside the transmission line gap to optimize the anode-cathode separation.

\section{TRANSMISSION LINE MODELING}

We study here three nested transmission line geometries specifically designed for HADES [15], the High Amperage Driver for Extreme States, located at the University of Rochester (shown in Fig. 2). The system has two modules.
Each module holds three cavities, capable of delivering up to $1 \mathrm{MA}$ each and stacked vertically. The two modules are connected using one set of nested transmission lines, sharing a common cathode. The top anode and the common cathode connect the top module to the load. The bottom anode and the common cathode connect the bottom module to the load using a set of post-hole convolutes.

To further simplify the design, we opted against magnetically insulated transmission lines [16]. If the electric field at the cathode stays below the electron emission field in a vacuum, taken to be $200 \mathrm{kV} / \mathrm{cm}$ for stainless steel, current losses across the gap are negligible. In practice, losses are small initially. However, they will become important when a plasma fills the anode cathode gap, a situation that is not considered in our simulations. Throughout this paper, we used a load inductance of $20 \mathrm{nH}$. This inductance is relatively high for standard pulsed-power loads (usually around $10 \mathrm{nH}$ as quoted in the introduction). Rather than looking at standard loads, this paper was more interested at looking at the maximum load that HADES could drive while reaching $1 \mathrm{MA}$. While such large inductances are unusual in fast Z-pinch studies, the inductance of a wound solenoid used to generate megagauss magnetic fields [17] can reach such values.

Using an optimal transmission line design [18] is problematic when transmission lines are nested, as the path to the load is longer for the bottom module. The first geometry, a set of wrapped transmission lines, balances the path length between the load and the top and bottom modules. Figure 2 shows the initial design of HADES wrapped transmission lines, while Fig. 3(a) shows the final design, where the anode-cathode distance keeps the electric

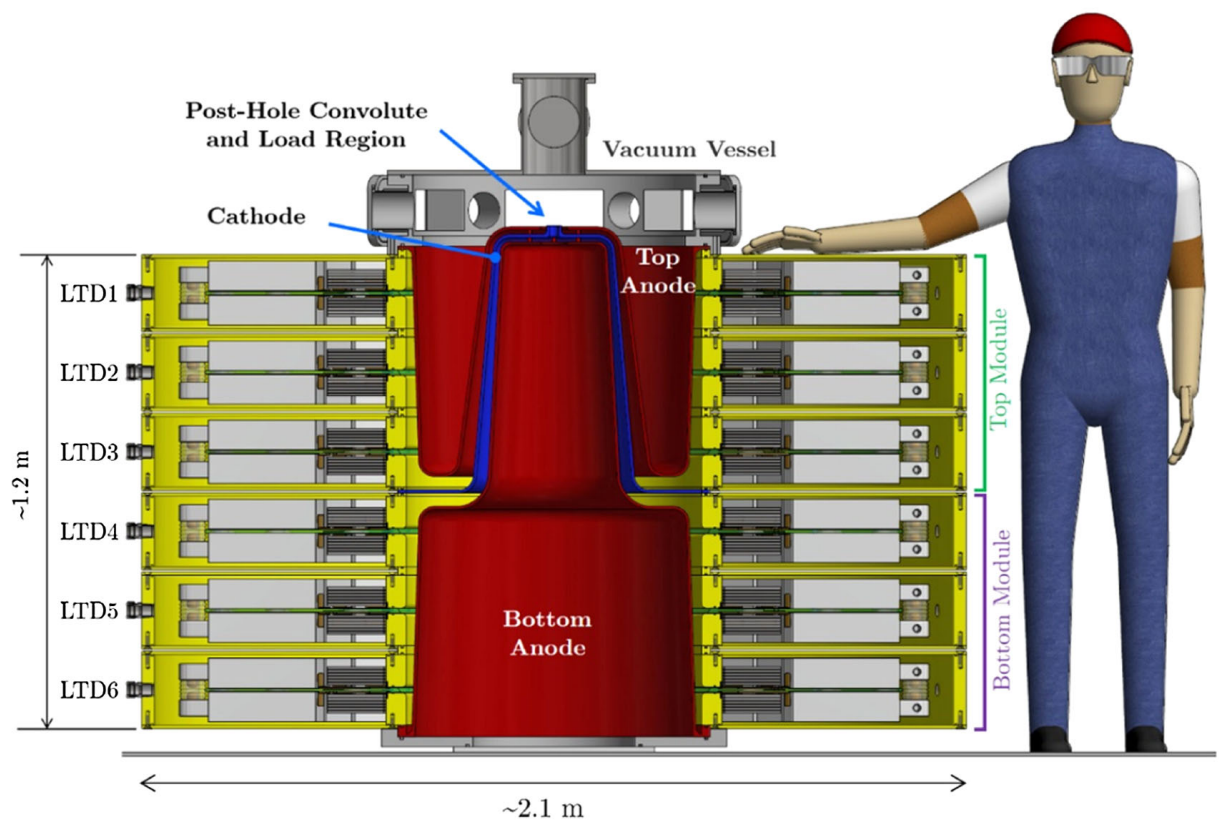

FIG. 2. A cutout view of HADES showing the location of the transmission lines (top and bottom anodes in red as well as the cathode in blue). The two modules are also shown. Each module has three identical cavities shown in Fig. 1 (yellow in this figure). 

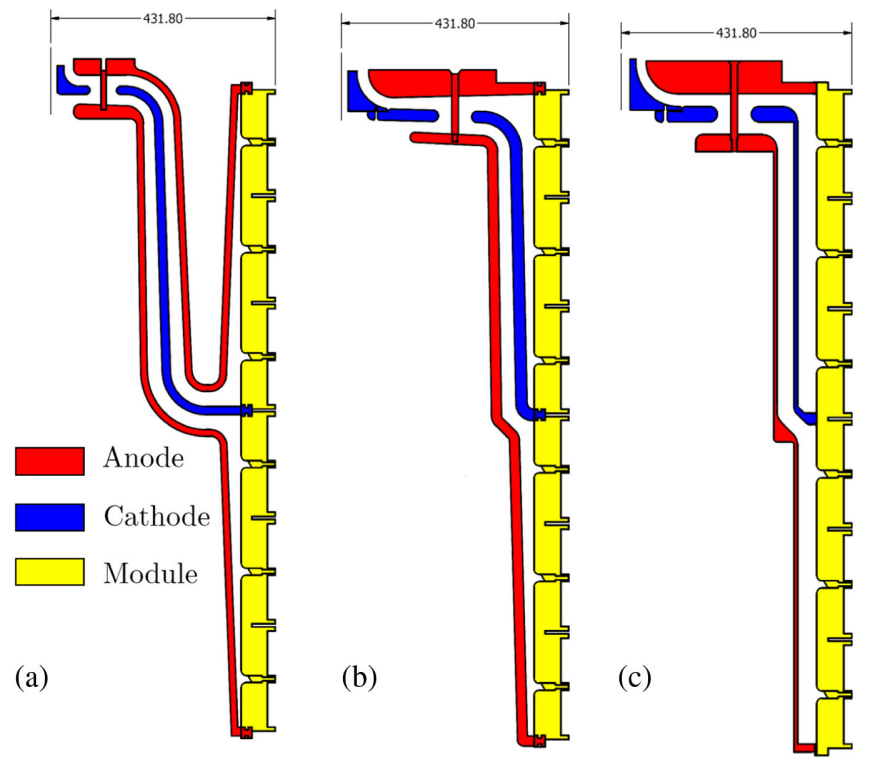

FIG. 3. The three different transmission lines studied in this paper: (a) wrapped, (b) conical, and (c) straight transmission lines.

field below the emission threshold of stainless steel. In this geometry, the current flows from top to bottom in the lower module, while it flows down and then up again in the upper module. Because the current path length is roughly the same, both sets of transmission lines have comparable inductances. This design keeps the currents and voltages even across all six cavities. Since they now deliver the same power, component wear will be identical for all cavities. However, the top transmission lines are longer than necessary.

It is possible to reduce the length of the upper transmission lines by taking a more direct path, as presented in Fig. 3(b). Now, the inductances of the top and bottom sets are different, leading to different loadings for the top and bottom modules. This conical design can also use deionized water to increase the capacitance of the transmission lines. The slope in the transmission lines was optimized to allow residual air bubbles to collect at the periphery of the top anode. The vacuum-water interface [not represented in Fig. 3(b)] is located near the load, past the post-hole convolute, to let the bubbles from the bottom transmission line reach the top anode.

Both designs need to be cast and then machined down to specifications. They both have relatively thick walls to minimize flexing during machining operations. It is possible to reduce the weight and cost of conical transmission lines by replacing the conical sections with straight, rolled tubes that would be welded to flat, horizontal plates, as shown in Fig. 3(c). The thick plates also add structural stiffness and partially correct the roundness deflections of the tube created by the rolling process. Using standard tube sizes (i.e., $23^{\prime \prime}$ and $26^{\prime \prime}$ ) further simplifies manufacturing. A slight slant could have been added to the horizontal plates to reduce the number of bubbles trapped in deionized water. However, this change was not implemented in our design study.

The current peak and rise time for all three geometries computed using circuit simulations [done with SPICE and shown in Figs. 4(a) and 4(c)] were compared to full 3D

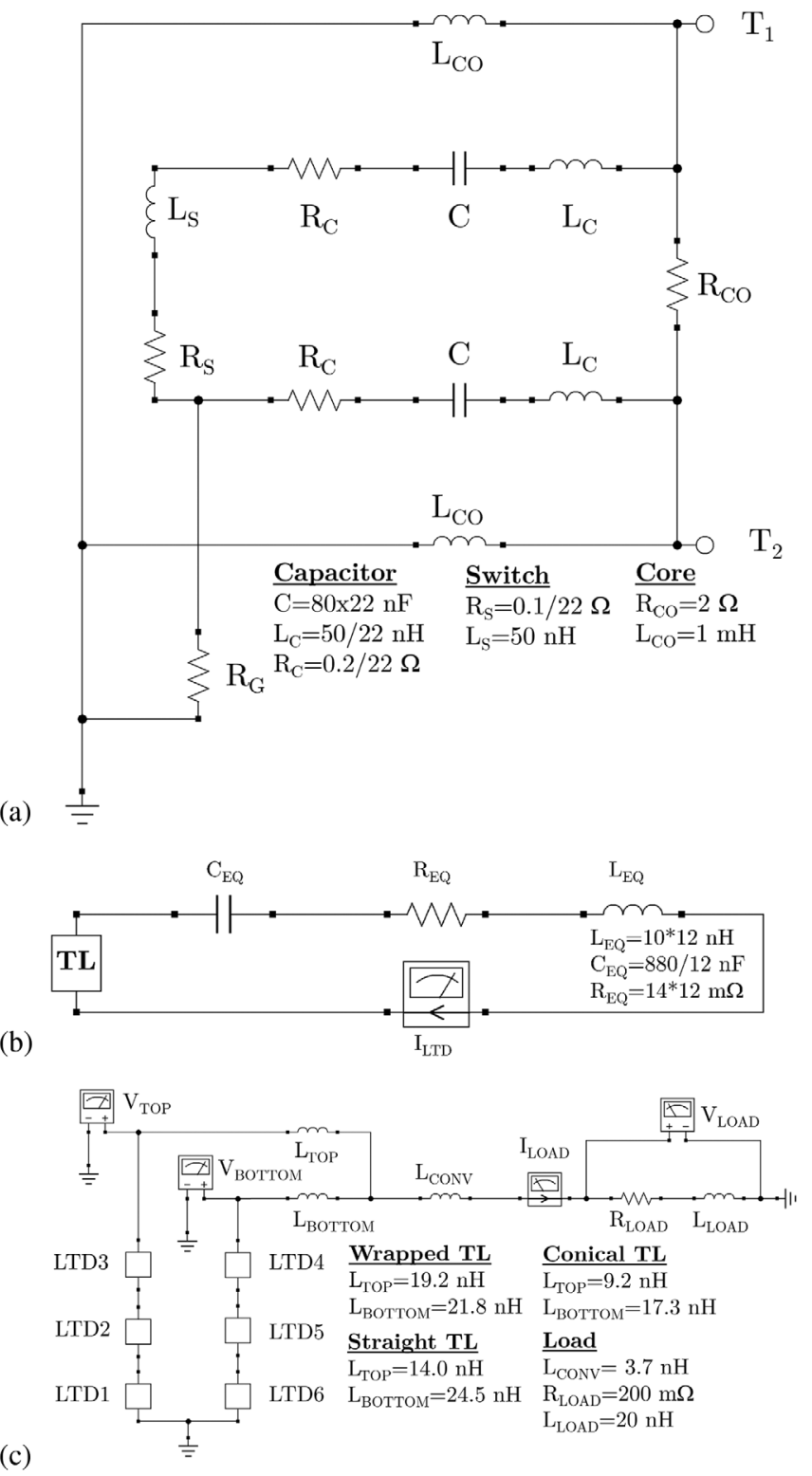

FIG. 4. (a) Equivalent RLC circuit for one cavity with 22 bricks used in SPICE simulations. $T_{1}$ and $T_{2}$ are the terminals used to connect one cavity to the next. $R_{G}$ is a $5 \mathrm{M} \Omega$ resistor, stabilizing the numerics. (b) Equivalent RLC circuit for one cavity used in COMSOL simulations. TL indicates the connection the transmission lines at a given height. (c) HADES equivalent RLC circuit. Each block (LTD1-6) uses the subcircuit shown in (a). The volt and amp meters are measuring the top (TOP) and bottom (BOTTOM) modules as well as the load (LOAD) voltages and current. The post-hole convolute inductance $\left(L_{\mathrm{CONV}}\right)$ was kept constant in all three designs. 


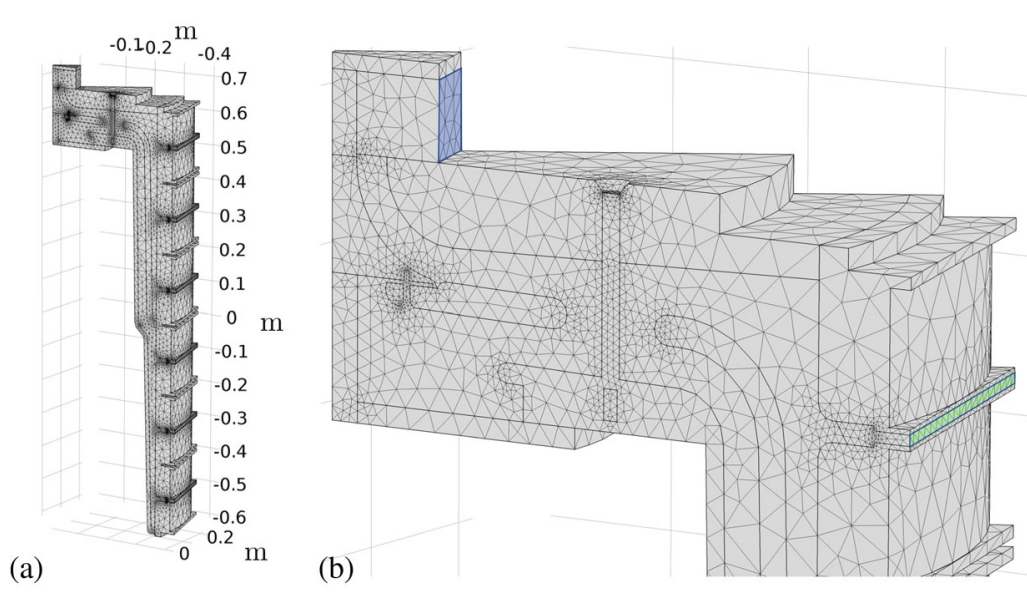

FIG. 5. (a) An overall view of the typical mesh used for the simulations. The mesh is generated directly from the CAD drawings. (b) Enlarging the mesh around the convolute region shows how the A-K gap of the cavity and the post-hole convolute are meshed. The light green region is the port where the LTD equivalent circuit shown in Fig. 4(b) is connected. The light blue region corresponds to the port connection of the load equivalent circuit. Several "vacuum" volumes were added under the load region to close the simulation domain, avoiding meshing the volume surrounded by the bottom anode.

electromagnetic simulations (using COMSOL). The 3D mesh for conical transmission lines is presented in Fig. 5(a). The meshes for the other two geometries are similar and not presented here. The mesh resolution is coarse enough to keep the computation time reasonable (several hours) yet fine enough so that no further insight was gained by increasing the resolution. The resolution does vary across the whole model to further reduce computational time. Regions with little curvature were resolved sufficiently to allow electromagnetic waves to propagate properly. The element density was an order of magnitude higher in regions with smaller radius of curvature [i.e., the anodecathode gap of each cavity, the post-hole convolute region shown in Fig. 5(b)]. The rf solver uses Maxwell's equations to compute implicitly wave propagation and displacement currents, while physical currents are derived from Ohm's law. We simulated all three cases using lossy transmission lines. The conductivity of the medium was increased to $1 \mathrm{mS} / \mathrm{m}$ to account for losses common in real transmission lines. These losses, caused by electron emission across the gap, also reduce reflections, commonly found in unmatched lossless transmission lines.

Ultimately, the study demonstrates that nested transmission lines can be used to connect modules together and allowed us to size the transmission lines to stay below the $200 \mathrm{kV} / \mathrm{cm}$ emission threshold. Therefore, all other components in the study are lump elements. Each cavity is modeled as an RLC circuit, shown in Fig. 4(b). The total inductance was increased by $4 \mathrm{nH}$ to account for stray inductances, such as the inductance of the strip lines, connecting the bricks to the inner ring.

A total of six of these circuits are connected across the A-K gap of each cavity. The connection of one of this circuits is shown in light green in Fig. 5(b). The connection, called a port in COMSOL, has an insulator with rectangular cross section surrounded by conductors. Another lump circuit is connected across the load region [port geometry shown in light blue in Fig. 5(b)]. This lump circuit is just a 20 -nH inductor coupled to the $0.2 \Omega$ load resistor. The resistor is matched to the complete circuit, including all six cavities as well as the load inductor, to limit current reversal on the capacitors. While real systems may not use a matched resistor, it is current practice to run simulations this way.

We also used a soft start to stabilize the electromagnetic solver using a variable inductor, $\mathrm{L}(\mathrm{t})$ as shown in Fig. 4(b) following the time evolution given by

$$
L(t)=\frac{1}{2} L_{\mathrm{START}}\left[1-\tanh \left(\frac{t}{t_{\text {rise }}}\right)\right]+L_{\mathrm{EQ}},
$$

where $L_{\mathrm{START}}=1 \mu \mathrm{H}, L_{\mathrm{EQ}}=10 \mathrm{nH}$, and $t_{\text {rise }}=10 \mathrm{~ns}$. Using a variable resistance would slow down simulations, as the system of hyperbolic differential equations becomes stiffer with an increased initial resistance. We supposed that all bricks triggered simultaneously, giving the highest possible electric field in the gap between the transmission lines.

\section{NUMERICAL SIMULATIONS}

The numerical simulations presented in this section cover the three geometries presented previously through four case studies: three using vacuum transmission lines and one using water lines. In all four cases, the capacitors were initially charged to a voltage equivalent to $\pm 95 \mathrm{kV}$ as it would be done during normal operations. We initially conducted COMSOL 2D simulations of the full system, including magnetic cores and strip lines, only replacing capacitors and switches with the lump model of Fig. 4(a). 

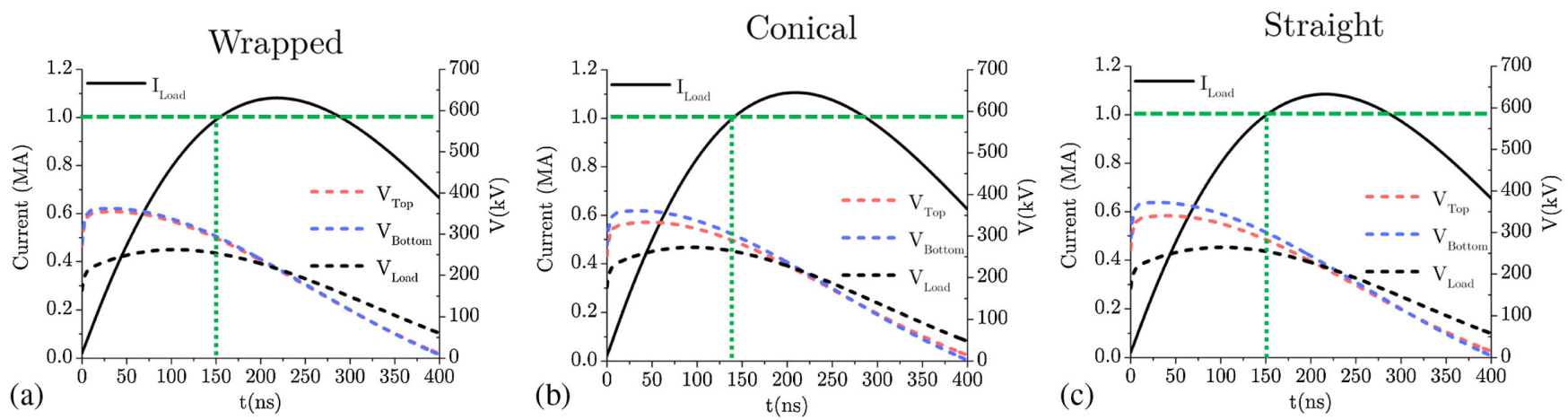

FIG. 6. SPICE simulations for (a) wrapped, (b) conical, and (c) straight transmission lines.

We found very little difference between the simulations with cores and full casing and the simulations with no cores and a truncated casing. However, two-dimensional simulations cannot capture the impact of the post-hole convolute geometry on the current rise. Consequently, three-dimensional simulations were necessary, but using a greatly simplified geometry, ignoring cores and the outer parts of the casings.

\section{A. Circuit simulations}

The transmission line and post-hole inductances were computed from the two-dimensional mechanical drawings using a simple integration technique. The lumped circuit simulations using SPICE shows that all transmission line geometries allow one to reach $1 \mathrm{MA}$ or more inside a $20-n H / 0.2-\Omega$ load. The wrapped transmission lines keep both modules balanced throughout the shot as seen in Fig. 6(a). The current reaches 1 MA in 153.7 ns. The maximum current value is $1.08 \mathrm{MA}$ at $218.2 \mathrm{~ns}$. Based on inductance calculations from Fig. 4(c), it was found that the conical transmission lines are the geometry with the smallest inductance. The current passes the 1-MA mark after $139.5 \mathrm{~ns}$ and reaches a peak of 1.11 MA in $209.2 \mathrm{~ns}$ [see Fig. 6(b)]. This is the best possible outcome of all three cases. As expected, the two modules see different impedances, and the module voltages are now slightly different (333 vs $362 \mathrm{kV}$ ). Compared to the wrapped transmission lines, the straight transmission lines give very similar results, reaching $1 \mathrm{MA}$ in $150.9 \mathrm{~ns}$ with a maximum current of $1.084 \mathrm{MA}$ at $211.7 \mathrm{~ns}$. However, the two modules are also out of balance, like for conical transmission lines.

Under the present modeling, all three designs are roughly equivalent. If our objective is to equilibrate the loading of both modules, then the wrapped transmission line design can be chosen, with very little penalty on the current rise and maximum value. If we want to achieve best performance, the conical design is the answer. Finally, if simplicity is the main concern, then the straight design is best.

\section{B. Electromagnetic simulations}

While both models used lump elements in both the drive and load, the current rise is different when using electromagnetic wave modeling. It was found that the soft start (with transition times on the order of $10 \mathrm{~ns}$ ) and mesh size (once optimized) had little impact on the total current rise. In all cases, electromagnetic transmission line simulations showed a slower current rise compared to circuit simulations. The voltage measured in the circuit simulations cannot be compared directly to the voltage in the electromagnetic simulations where voltage monitors see a portion of the transmission line voltage. Yet, we found that the current evolution is overall consistent for both wrapped [shown in Fig. 7(a)] and conical [shown in Fig. 7(b)] transmission lines.

The wrapped transmission lines worked again as designed, keeping almost the same voltage across both modules throughout the discharge. The current reaches 1.05 MA in $268.5 \mathrm{~ns}$ (instead of 1.08 MA in 218.2 ns). Figure 7(a) shows that the currents in the top three cavities are equal, as would be the case in a real module. The currents in the bottom three cavities are also equal. Since cavities are in series inside a module, the same current must flow through all of them. COMsOL computes the current in the RLC circuit of load and cavities from the I-V characteristic of the transmission line ports [labeled TL in Fig. 4(b)]. So, current conservation throughout all cavity and load circuits helps validate the simulations. When the transmission lines have different lengths, the modules deliver different currents. The current in the conical transmission lines is shown in Fig. 7(b) and in Fig. 7(c) for the straight transmission lines. The conical lines reach a total of $1.07 \mathrm{MA}$ in $269.5 \mathrm{~ns}$, while the straight transmission lines deliver $1 \mathrm{MA}$ in 287.4 ns. Figure 8 highlights the differences in load currents between COMSOL and RLC circuit simulations for all three cases.

Full electromagnetic simulations were necessary to evaluate the electric fields across the gap and optimize the transmission line gap to keep the field below 

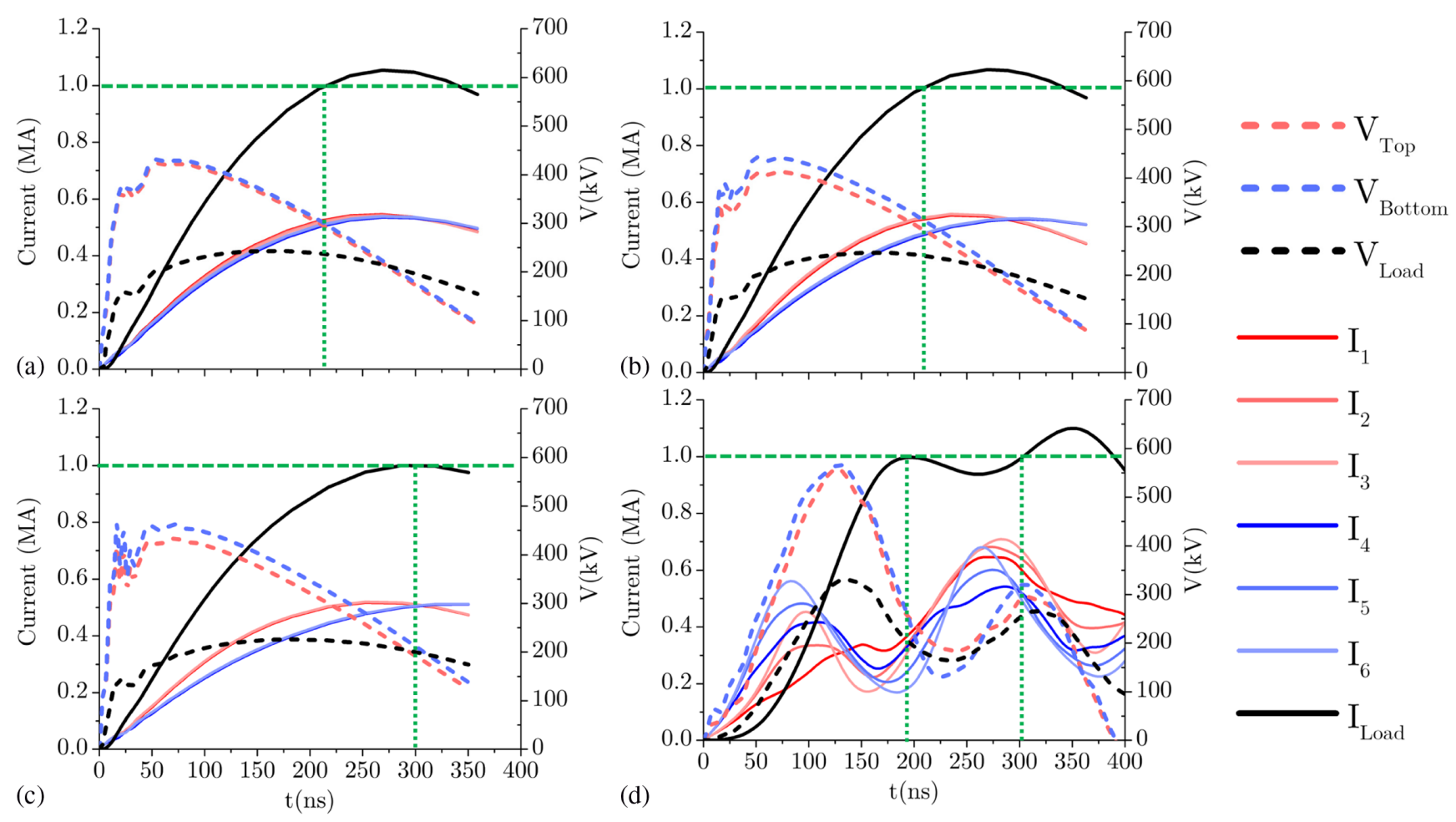

FIG. 7. Time evolution of the current for a $20 \mathrm{nH} / 0.2 \Omega$ load with (a) wrapped, (b) conical, (c) straight, and (d) conical with water transmission lines using COMSOL. The currents $I_{1}$ to $I_{6}$ are the current in the cavities 1-6.

$200 \mathrm{kV} / \mathrm{cm}$. The optimization procedure decreased the anode-cathode gap until the field strength on the cathode remained below the prescribed threshold. Figure 9 shows the field strength throughout the transmission lines when the discharge voltage is maximum.

This study also considered water transmission lines. Since both wrapped and straight transmission lines can trap air bubbles, we presented here only conical transmission lines. The current waveform at the load, shown in Fig. 7(d), has a double hump with a current rise time of $197 \mathrm{~ns}$ to first peak, at $1 \mathrm{MA}$. The second peak is reached after $350.2 \mathrm{~ns}$, at 1.1 MA. While this option was not considered in the initial design, we still find it very attractive, and it should be explored further. However, the introduction of water in the transmission lines also increases the parasitic capacitance of the lines, and displacement currents can rival conduction currents flowing along the transmission line, leading to different currents in each cavity, as shown in Fig. 7(d). Because the current rise time is shorter when water is present, the voltage at the load is also larger. This indicates that the anode-cathode gap should be increased in the final design.
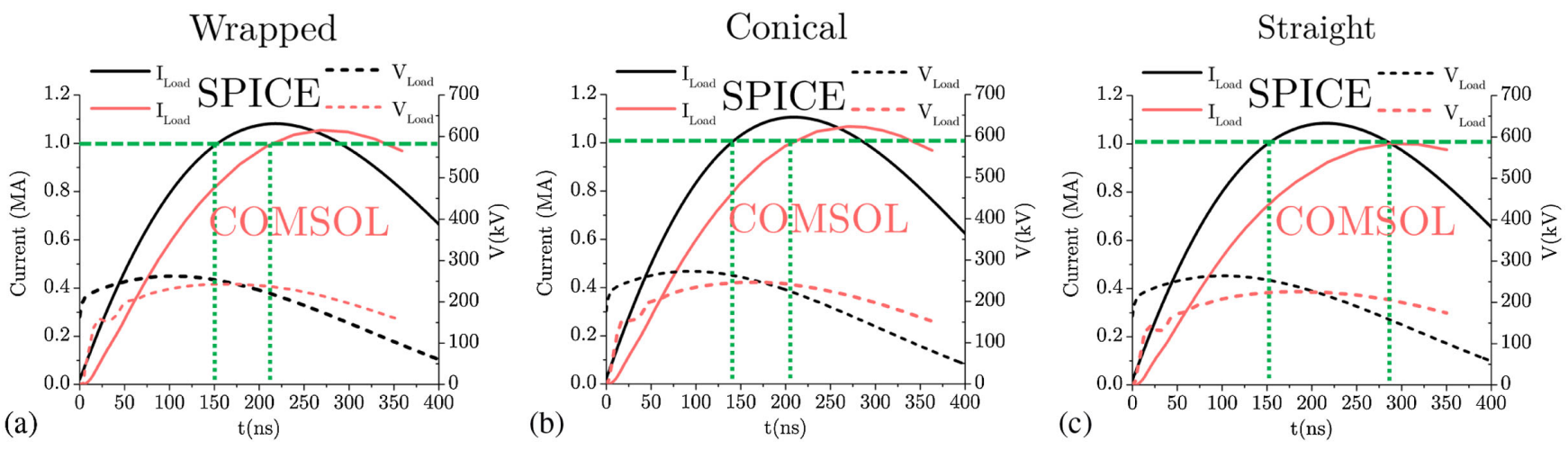

FIG. 8. Comparison between circuit and electromagnetic simulations for (a) wrapped, (b) conical, and (c) straight transmission lines. 


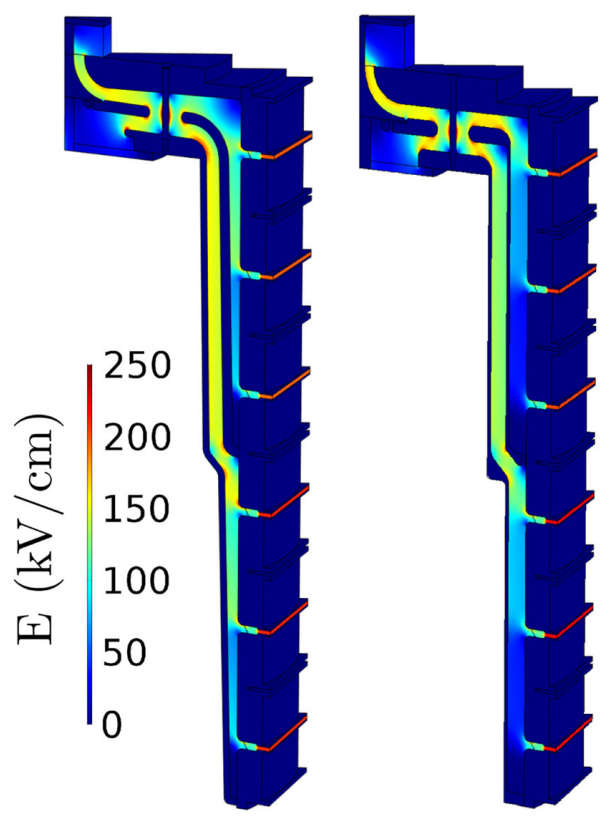

FIG. 9. Electric field strength computed by COMSOL for the conical (left) and straight (right) transmission lines. The view is the same as the view in Fig. 5.

\section{CONCLUSIONS}

This study showed how nested transmission lines can be used to add currents from separate modules in MA-class LTD systems. This implementation simply wraps each cavity around a current adder assembly, found in many pulsed-power drivers (e.g., COBRA [19] and Z [20]). This design bypasses the usual long module transmission lines, greatly decreasing the inductance of the system. While this design does not scale efficiently to more than two modules, it works well for MA-class pulsed-power drivers. The three designs explored here have distinct advantages. Conical transmission lines are optimized to connect two modules together. However, their fabrication cost has been shown to be relatively high. Wrapped transmission lines are best for balancing power coupling. Straight transmission lines are the simplest and easiest to manufacture.

\section{ACKNOWLEDGMENTS}

This research was supported in part by the NSF/DOE Partnership in Basic Plasma Science and Engineering via a DOE Office of Science, Fusion Energy Sciences Grant No. DE-SC0016252, the NSF MRI Grant No. PHY1725178, the NSF CAREER Grant No. PHY-1943939, the University of Rochester, and the PPPL DOE Contract No. DE-AC02-09CH11466.

[1] A. A. Kim, M. G. Mazarakis, V. A. Sinebryukhov, B. M. Kovalchuk, V. A. Visir, S. N. Volkov, F. Bayol,
A. N. Bastrikov, V. G. Durakov, S. V. Frolov, V. M. Alexeenko, D. H. McDaniel, W. E. Fowler, K. LeChien, C. Olson, W. A. Stygar, K. W. Struve, J. Porter, and R. M. Gilgenbach, Development and tests of fast 1-MA linear transformer driver stages, Phys. Rev. Accel. Beams 12, 050402 (2009).

[2] M. G. Mazarakis, W.E. Fowler, A. A. Kim, V. A. Sinebryukhov, S. T. Rogowski, R. A. Sharpe, D. H. McDaniel, C. L. Olson, J. L. Porter, K. W. Struve, W. A. Stygar, and J. R. Woodworth, High current, 0.5-MA, fast, 100-ns, linear transformer driver experiments, Phys. Rev. Accel. Beams 12, 050401 (2009).

[3] R. D. McBride, W. A. Stygar, M. E. Cuneo, D. B. Sinars, M. G. Mazarakis, J. J. Leckbee et al., A primer on pulsed power and linear transformer drivers for high energy density physics applications, IEEE Trans. Plasma Sci. 46, 3928 (2018).

[4] F. Lassalle, C. Mangeant, B. Roques, A. Georges, A. Loyen, J.F. Cambonie, S. Laspalles, D. Cadars, G. Rodriguez, J. M. Delchie, P. Combes, T. Chanconie, and J. Saves, in Proceedings of the 14th Symposium on High Current Electronics (14th SHCE), Tomsk, Russia (2006).

[5] S. C. Bott, D. M. Haas, R. E. Madden, U. Ueda, Y. Eshaq, and G. Collins IV, K. Gunasekera, D. Mariscal, J. Peebles, F. N. Beg, M. Mazarakis, K. Struve, and R. Sharpe, 250 kA compact linear transformer driver for wire array z -pinch loads, Phys. Rev. Accel. Beams 14, 050401 (2011).

[6] R. M. Gilgenbach, M. R. Gomez, J. C. Zier, W. W. Tang, D. M. French, Y. Y. Lau, M. G. Mazarakis, M. E. Cuneo, M. D. Johnston, B. V. Oliver, T. A. Mehlhorn, A. A. Kim, and V. A. Sinebryukhov, MAIZE: a 1 MA LTD-driven Z-pinch at The University of Michigan, AIP Conf. Proc. 1088, 259 (2009).

[7] W. A. Stygar, M. E. Cuneo, D. I. Headley, H. C. Ives, R. J. Leeper, M. G. Mazarakis, C. L. Olson, J. L. Porter, T. C. Wagoner, and J.R. Woodworth, Conceptual designs of two petawatt-class pulsed-power accelerators for highenergy-density-physics experiments, Phys. Rev. Accel. Beams 18, 110401 (2015).

[8] J. D. Douglass et al., $100 \mathrm{GW}$ linear transformer driver cavity: Design, simulations, and performance, Phys. Rev. Accel. Beams 21, 120401 (2018).

[9] S. A. Slutz, M. C. Herrmann, R. A. Vesey, A. B. Sefkow, D. B. Sinars, D. C. Rovang, K. J. Peterson, and M.E. Cuneo, Pulsed-power-driven cylindrical liner implosions of laser preheated fuel magnetized with an axial field, Phys. Plasmas 17, 056303 (2010).

[10] D. D. Ryutov, M. S. Derzon, and M. K. Matzen, The physics of fast $Z$ pinches, Rev. Mod. Phys. 72, 167 (2000).

[11] W. A. Stygar, M. E. Cuneo, D. I. Headley, H. C. Ives, R. J. Leeper, M. G. Mazarakis, C. L. Olson, J. L. Porter, T. C. Wagoner, and J.R. Woodworth, Architecture of petawattclass Z-pinch accelerators, Phys. Rev. Accel. Beams 10, 030401 (2007).

[12] D. D. Ryutov, R.P. Drake, J. Kane, E. Liang, B. A. Remington, and W. M. Wood-Vasey, Similarity criteria for the laboratory simulation of supernova hydrodynamics, Astron. J. 518, 821 (1999).

[13] T. A. Shelkovenko, I. N. Tilikin, G. V. Ivanenkov, W. Stepniewski, A. R. Mingaleev, V. M. Romanova, 
A. V. Agafonov, A. D. Cahill, C. L. Hoyt, P. A. Gourdain, D. A. Hammer, and S.A. Pikuz, Dynamics of hybrid X-pinches, Plasma Phys. Rep. 41, 52 (2015).

[14] G. F. Swadling, S. V. Lebedev, G. N. Hall, S. Patankar, N. H. Stewart, R. A. Smith, A. J. Harvey-Thompson, G. C. Burdiak, P. de Grouchy, J. Skidmore, L. Suttle, F. Suzuki-Vidal, S. N. Bland, K. H. Kwek, L. Pickworth, M. Bennett, J. D. Hare, W. Rozmus, and J. Yuan, Diagnosing collisions of magnetized, high energy density plasma flows using a combination of collective Thomson scattering, Faraday rotation, and interferometry (invited), Rev. Sci. Instrum. 85, 11 E502 (2014).

[15] P.-A. Gourdain, M. Evans, B. Foy, D. Mager, R. McBride, and R. Spielman, HADES: A high amperage driver for extreme states, arXiv:1705.04411.

[16] C. W. Mendel, Jr. and S. E. Rosenthal, Modeling magnetically insulated devices using flow impedance, Phys. Plasmas 2, 1332 (1995).

[17] P.-A. Gourdain, G. Brent, J. B. Greenly, D. A. Hammer, and R. V. Shapovalov, The generation of mega-gauss fields on the Cornell beam research accelerator, Rev. Sci. Instrum. 89, 095102 (2018).

[18] O. A. Hurricane, Optimized minimal inductance transmission line configuration for Z-pinch experiments, J. Appl. Phys. 95, 4503 (2004).

[19] J. B. Greenly, J. D. Douglass, D. A. Hammer, B. R. Kusse, S. C. Glidden, and H.D. Sanders, A 1MA, variable risetime pulse generator for high energy density plasma research, Rev. Sci. Instrum. 79, 073501 (2008).

[20] D. H. McDaniel, M. G. Mazarakis, D. E. Bliss, J. M. Elizondo, H. C. Harjes, H. C. Ives III, D. L. Kitterman, J. E. Maenchen, T. D. Pointon, S. E. Rosenthal, D. L. Smith, K. W. Struve, W. A. Stygar, E. A. Weinbrecht, D. L. Johnson, and J.P. Corley, in Proceedings of the 5th International Conference on Dense Z-Pinches, edited by C. Deeney, N. R. Pereira, and J. Davis (AIP, Melville, NY, 2003), No. 651, p. 23.

Correction: An NSF CAREER grant number was missing in the Acknowledgments section and has been inserted. 